\title{
The intersection of COVID-19 and public health care in Canada: What does the future hold for the surgical patients and health care workers left behind?
}

The views expressed in this editorial are those of the author and do not necessarily reflect the position of the Canadian Medical Association or its subsidiaries.

$t$ is with great sadness that we write another editorial on the impact of COVID-19. All health care providers were desperately hoping to bring in a new year full of optimism, promise and, most importantly, an era free of COVID-19 concerns. Of course, this hasn't been the case. ${ }^{1,2}$ As we hover around the 2-year anniversary of the pandemic, it is worth taking time to reflect on its impact both on health care workers and on the health care system as a whole. ${ }^{3}$

In the not-so-distant future, one wonders if COVID19 will be retrospectively viewed as the straw that broke the Canadian health care system's back. More specifically, in many of our provinces, surgical systems have traditionally functioned at nearly $100 \%$ capacity (beds, procedures, staffing, patient throughput). Unfortunately, this reality has left little to no room for "normal" surgical care in the context of a system-wide need for medical surge capacity and the resulting redirection of health care resources away from surgical operations to fight the COVID-19 pandemic. More to the point, surgical care for patients with deteriorating joints, painful extremities, debilitating hernias and abdominal pains, profound reconstruction needs, and even life-threatening cancers has been put on hold (at best) in favour of focusing on the relentless and unremitting pulses of SARS-CoV-2 infections. To be clear, this reality has overwhelmed nearly all health care workers' ability to provide adequate acute and urgent care to our local populations. ${ }^{4}$ This is no more obvious than within the surgical domain. Despite working as hard as possible, surgical staff have witnessed decrements in patient care, alterations in the definitions of the "standard of care," and an inability to expeditiously move toward normal health care delivery between COVID waves. The ability to "catch up" on surgical procedure volumes after the pandemic ceases also seems unlikely, given the tremendous increase in the number of surgeons, nurses, respiratory therapists, anesthesiologists and support staff that would be required to complete such an endeavour. Where would these folks come from? How would the associated financial costs be covered? From where would the caregiver will to do more than previously possible arise?

We must also recognize the tremendous psychological impact of COVID-19 on the health care workers. As our military colleagues know, the public health care system has been engaged in "combat operations" for nearly 2 years. The deterioration in surgical care for non-COVID-19 patients has been difficult to "unsee" and is a reality that has led to both burnout and moral injury. Not surprisingly, a drift out of Canadian health care jobs by all types of workers (nurses, physicians, support staff) is now increasingly common in the form of general attrition, early retirement, and migrations to jobs in the United States. This exodus has not been helped by the anger among our patient populations who are desperately unable to access timely surgical care. Some of this rage has led to protests at numerous hospitals across the country (with some startling confrontations), patient aggression toward health care workers within clinics and hospitals as potential perpetrators of harm, and frequent discussions among front-line providers of feeling abandoned by the managerial and political leadership within our health care systems. In some provinces, this has been compounded by massive cuts to physician and system budgets contextualized within public confrontations with government, supply-chainrelated scarcity of essential surgical and medical equipment, and the general feeling among health care workers that there is no clear pathway out of the tremendously large patient surgical care deficit.

The reality is that health care in Canada has always been used as both a powerful political tool and potential electoral liability. Our system is clearly underresourced within the current ongoing COVID-19 crisis, as it has lost the ability to adequately care for many vaccinated Canadians who require both life-saving and quality-oflife-sustaining operations. The front-line health care workers who have been witness to the realities of both population-level and patient-level harm will likely never be the same. It becomes natural to question one's own 
future within this system, as well as the future of the Canadian public health care systems.

To all health care workers who continue to arrive at their clinics, hospitals and client homes full of resolve, caring and hope, we can't thank you enough. You are the glue that holds our surgical system together. We remain in awe of you. To all workers who are struggling, depressed and pessimistic about the future of Canadian health care, we stand with you. We support you. The moral injury experienced by your witness of our collective inability to prevent patient harm will be a lasting legacy of the pandemic. With some trepidation, we remain hopeful, however, that we can all contribute to a postpandemic way forward from within the clinical trenches in a manner that is heard by both elected and appointed leadership. This pathway must be inclusive, comprehensive, efficient and compassionate in its beneficence for all of our surgical patients.

Chad G. Ball, MD, MSc; Paul T. Engels, MD; Sean Gregg, MD; Edward J. Harvey, MD.

Affiliations: From the Department of Surgery, University of Calgary, Calgary, Alta. (Ball); City View Surgical Associates, Red Deer, Alta. (Gregg); the Department of Surgery, McMaster University, Hamilton, Ont. (Engels); and the Department of Surgery, McGill University, Montreal, Que. (Harvey).
Competing interests: E.J. Harvey is the cofounder and head of medical innovation of NXTSens Inc.; the cofounder and chief medical officer of MY01 Inc., and Sensia Diagnostics Inc.; and the cofounder and director of Strathera Inc. He receives institutional support from J \& J DePuy Synthes, Stryker, MY01, and Zimmer. No other competing interests were declared.

Content licence: This is an Open Access article distributed in accordance with the terms of the Creative Commons Attribution (CC BYNC-ND 4.0) licence, which permits use, distribution and reproduction in any medium, provided that the original publication is properly cited, the use is noncommercial (i.e., research or educational use), and no modifications or adaptations are made. See: https://creativecommons. org/licenses/by-nc-nd/4.0/

Cite as: Can J Surg 2022 January 27; 65(1). doi:10.1503/cjs.000422

\section{References}

1. Centers for Disease Control and Prevention. Omicron variant. CDC; 2021. Available: https://www.cdc.gov/coronavirus/2019-ncov/ index.html (accessed 2021 Dec. 25).

2. Ball CG. COVID-19: a time of crisis, but also of surgical opportunity and optimism. Can F Surg 2020;63:164-5.

3. Ball CG. Leadership during the COVID-19 crisis and beyond. Can 7 Surg 2020;63:E370-E371.

4. Finley C, Prashad A, Camuso N, et al. Guidance for management of cancer surgery during the COVID-19 pandemic. Can 7 Surg 2020;63:2-4. 